ESAIM: PROCEEDINGS AND SURVEYS, October 2015, Vol. 51, p. 60-73

A. Garivier et al, Editors

\title{
LEARNING ABOUT CRITICAL PHENOMENA FROM SCRIBBLES AND SANDPILES*
}

\author{
ADRIEN KASSEL ${ }^{1}$
}

\begin{abstract}
This text grew out of the notes for a talk given at the Journées MAS 2014. We give a concise report on our recent work on loop-erased random walk and related processes (spanning trees, cycle-rooted spanning forests, and sandpiles) on planar lattices and on graphs embedded in Riemannian surfaces in the limit of small meshsize.

Résumé. Ce texte est issu des notes d'un exposé donné aux Journées MAS 2014. Nous présentons une synthèse de nos travaux récents sur la marche aléatoire à boucles effacées et certains processus reliés (tels les arbres couvrants, les forêts couvrantes d'unicycles, le tas de sable abélien) sur les réseaux planaires et sur les graphes plongés dans les surfaces riemanniennes dans la limite où la maille tend vers zéro.
\end{abstract}

\section{INTRODUCTION}

In equilibrium statistical physics, a system is said to be in a critical state when it exhibits long-range correlations and some form of scale invariance. This self-similarity at different length-scales is often related to the fact that correlations of observables at two different locations decay as power laws with the distance. The corresponding exponents, called critical exponents, are thought to be universal, that is, independent of the microscopic structure of the system. Examples of such critical behaviour arise in the study of models of phase transitions, like the model of percolation or the Ising model. There, one considers a parameter-dependent system which undergoes a phase transition for a particular value of the parameter called the critical point; the latter is generally dependent on the microscopic details of the system. When the parameter reaches the critical point, the system is shown to be in a critical state.

The concept of self-organised criticality was proposed in [5] to describe off-equilibrium systems of interacting particles that are dynamically driven towards a state which exhibits properties that are characteristic of a critical state, although this state does not appear to be related to the existence of a phase transition in the sense described above. It has been argued that this mechanism is ubiquitous in nature and that it accounts for many of the fractal features, in time or space, observed in phenomena as varied as earthquakes, forest fires, river networks, luminosity of stars, neural activity, and so on $[4,51]$.

In order to reproduce behaviours found in empirical data collected in the observation of self-organised critical phenomena, and to explain possible mechanisms leading to them, statistical physicists introduced several simple toy-models. We shall focus on two of them. Understanding their randomness and geometry may be seen as a motivation for the mathematical results we present here.

* The author acknowledges the hospitality of ENS Paris, Brown University, MSRI in Berkeley, Microsoft Research Redmond, and Université Paris Sud. Principal support was provided by a French doctoral grant. Additional support was provided by the Fulbright Foundation and the Fondation Sciences Mathématiques de Paris

${ }^{1}$ ETH, Departement Mathematik, Rämistrasse 101, 8092 Zürich, Switzerland; e-mail: adrien.kassel@math.ethz.ch

(C) EDP Sciences, SMAI 2015 
Let $\mathcal{G}=(V, E)$ be a finite connected graph with vertex-set $V$ and edge-set $E$, and define the Laplacian of $\mathcal{G}$ to be the $V$-indexed matrix $\Delta=D-A$, where $D$ is the degree matrix and $A$ the adjacency matrix.

The first model we introduce is a dynamical version of the loop-erasure of the random walk introduced by Lawler in [42]. Consider a simple nearest-neighbor random walk on $\mathcal{G}$, running in discrete time, whose steps are governed by the transition matrix $P=D^{-1} A$. As the walk evolves, we keep in memory the trajectory in the following way. Each time the trajectory loops, we erase the loop, keeping in memory the initial part of the trajectory, and continue the random walk from the tip of the remaining curve. If $\mathcal{G}$ is an infinite lattice (like the two-dimensional square grid $\mathbb{Z}^{2}$ ), or if $\mathcal{G}$ is a very large subgraph of such an infinite lattice, the loops created and erased in this process give rise to a simple model of a self-organized critical measure, as can be checked by performing some experiments on a computer. In such an experiment, we can keep track of the loops being erased and plot their lengths as a function of time, as in Figure 1. The distribution of these lengths as well as that of other observables is seen to have the kind of heavy power-law tails characteristic of criticality. This process has been studied in [1] where predictions on the critical exponents were made, which were later backed by rigorous computations of exponents for intersections of random walks [35, 43]. If we define the looping rate to be the rate $\rho$ at which loops are created, the mean value of the length distribution is $1 / \rho$. We will say more about how to compute $\rho$ later on, but let us first introduce the second model.
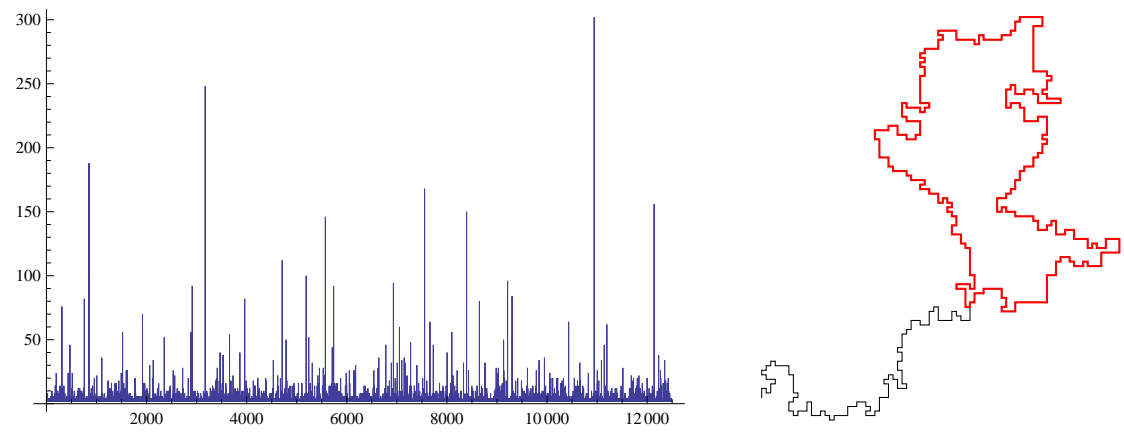

FiguRE 1. Left: lengths of the loops erased as a function of time; Right: the loop-erased random walk at a time where a very long loop (in red) is erased

The second model which shall interest us is the abelian sandpile model of Dhar [15], and it is closely related to the previous one. We start by some definitions. Assume for now that $\mathcal{G}$ is finite. Single out a vertex $s$ and call it the sink (the other vertices $W=V \backslash\{s\}$ are called internal vertices). Denote by $\Delta_{\widehat{s}}$ the sub-matrix of $\Delta$ obtained by erasing out the row and column indexed by $s$. A configuration of sand (or sandpile) is a nonnegative integer-valued height-function $\sigma$ on the internal vertices (heuristically, one may think of these values as the slope-angles of a real-world pile of sand, although the model is of course not intended as a realistic model of piles of sand). A sandpile configuration is unstable at an internal vertex if the height exceeds the threshold equal to the vertex degree (note that in the physics literature, the convention shifts all values by +1 ). The configuration at that vertex may then topple, meaning that one grain of sand is sent to each of the neighbors, leaving the vertex with a new amount of sand grains equal to the previous amount minus the vertex degree. Note that a toppling at a vertex $x$ corresponds to subtracting from the height vector, the row of $\Delta_{\widehat{s}}$ indexed by $x$, i.e. to the operation $\sigma \mapsto \sigma(\cdot)-\Delta_{\widehat{s}}(x, \cdot)$. Because of the presence of the sink, which absorbs any grain of sand falling in it, the system is dissipative and it is easy to see that any unstable configuration can be stabilized by performing a sequence of topplings until no unstable vertex remains. It is an instructive exercise to show that the resulting stable configuration does not depend on the order in which these successive topplings are performed (this is the abelian property, see [24] for a proof). A sequence of topplings leading to stabilization, as in the example of Figure 2, is called an avalanche.

The abelian sandpile model is defined as a Markov chain in discrete time whose steps consists in adding a grain of sand at a random internal vertex followed by stabilization. The recurrent states of this Markov chain are 
called the recurrent configurations. A remarkable property is that the unique stationary measure is uniform on the set of recurrent configurations. One way of proving it is by showing that the set of recurrent configurations endowed with the operation of point-wise addition followed by stabilization has the structure of an abelian group, called the sandpile group [9], isomorphic to $\mathbb{Z}^{W} / \Delta \widehat{\mathbb{Z}}^{W}$; the Markov chain is thus a random walk on a regular graph, the Cayley graph coming from a presentation of this group. By computing the invariant factors of this abelian group using the Smith normal form, the order of the sandpile group is easily seen to be det $\Delta \widehat{s}^{\text {, }}$ which, by the matrix-tree theorem, is the number of spanning trees of $\mathcal{G}$. There are thus as many recurrent configurations of sand as spanning trees of $\mathcal{G}$. Several interesting (but non local) bijections are known between recurrent sand piles and spanning trees, see e.g. [8,14].

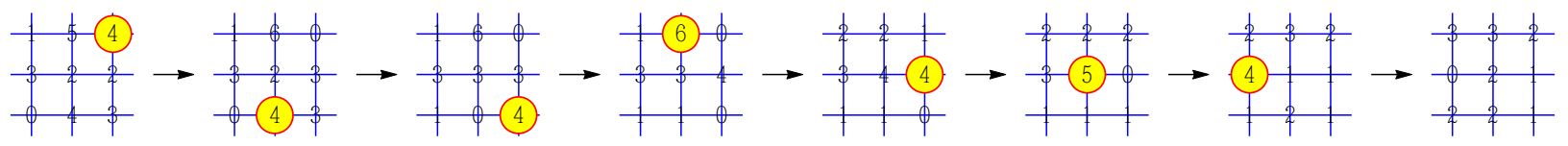

FiguRE 2. Example of a stabilization sequence on a $3 \times 3$ square grid where the sink (not drawn but represented by the region outside the grid) is connected to all boundary edges. At each vertex, the integer number represents the sandpile height and, at each step, the circled vertex is the one which is about to topple. The final configuration is stable.

If $\mathcal{G}$ is infinite, the model may be defined by taking the thermodynamic limit of the model on an exhausting sequence of finite subgraphs. This definition is independent of the exhausting sequence whenever each component of the uniform spanning forest in $\mathcal{G}$ has one end [27] which is the case for $\mathbb{Z}^{2}$-periodic connected planar graphs [6].

The sandpile density $\bar{\sigma}$ is defined as the amount of sand per vertex. This a priori random quantity turns out to be almost surely constant for an infinite periodic planar lattice due to a law of large number. By a slight abuse of terminology, we call sandpile density the expectation of the amount of sand per vertex for a finite graph (this deterministic quantity converges to the sandpile density when the infinite lattice is exhausted by a sequence of finite subgraphs).

The sandpile density may loosely be thought of as a critical parameter which measures the competition effect between entropy (number of configurations) and energy (total amount of sand) and which depends on the microscopic structure of the lattice. Although this last intuition is compelling, it is not quite right as we now explain. A related model of phase transition is the fixed energy sandpile [61]. In this model, defined on an infinite lattice, the system is conservative (no sink) and an initial random Poissonian configuration is sampled with a prescribed density. It is widely believed and backed numerically that there is a phase transition in the density-parameter, between a low density phase where stabilization almost surely occurs, and a high density phase where almost surely the configuration remains unstable forever. It has been argued heuristically that the critical density for the fixed energy model should coincide with $\bar{\sigma}$, and on the square lattice this is indeed numerically the case up to a $0.01 \%$ error. However, Fey, Levine, and Wilson [19] numerically disproved this conjecture and showed that the two values are in fact distinct (see [28] however).

\section{Planar lattices}

In this section we explain how to compute the sandpile density and looping rate for any planar $\mathbb{Z}^{2}$-periodic lattice ${ }^{1}$ and discuss related computations. In the case of the square lattice, it was first conjectured by Grassberger that $\bar{\sigma}=17 / 8$. The proof of Grassberger's conjecture appeared independently in $[12,39,57]$ almost two decades later. Kenyon and Wilson furthermore computed $\bar{\sigma}$ for the triangular and hexagonal lattices (in a

\footnotetext{
${ }^{1}$ To avoid any confusion, let us stress that we here use the word "lattice" in its widely accepted sense in statistical physics and not in the mathematical sense of a discrete subgroup of $\mathbb{R}^{2}$. Here, by a $\mathbb{Z}^{2}$-periodic lattice, we mean an infinite connected planar graph invariant under a rank-2 group of Euclidean translations.
} 
paper [39] which goes far beyond proving this conjecture). The proof we present here, obtained jointly with David Wilson [33], recovers these results in a much simpler way which also readily works for any periodic planar lattice. Our proof relies on counting certain subgraphs, which we now introduce.

\subsection{Counting spanning unicycles of planar graphs}

Counting spanning forests can be achieved by a corollary of the classical matrix-tree theorem. Although the matrix tree theorem is widely known and used, this corollary seems to have been less popular so far. It first appeared in Liu and Chow [48], and a simplified account was given by Myrvold [53]. In [33], building on this formula, we obtained with David Wilson that the number $F_{2}$ of two-component spanning forests is related to the number $F_{1}$ of spanning trees by the identity

$$
\frac{F_{2}}{F_{1}}=\sum_{u \sim v}\left(A_{u, v}^{(s)}-A_{v, u}^{(s)}\right)^{2}+A_{u, v}^{(s)} A_{v, u}^{(s)},
$$

where $A_{u, v}^{(s)}=G_{u, u}^{(s)}-G_{u, v}^{(s)}$ and $G^{(s)}$ is the inverse of $\Delta_{\widehat{s}}$ (called the Green function with Dirichlet boundary conditions at $s$ ) and the sum is over all pairs of neighboring vertices.

As it turns out, the values of $\bar{\sigma}$ and $\rho$ are related to the ratio $U_{1} / F_{1}$ where $U_{1}$ is the number of spanning unicycles. No exact formula for the number of unicycles is known to exist for general graphs ${ }^{2}$, but for planar graphs, this number is simply the number of two-component spanning forests for the dual graph.

\subsection{Loop-erased random walk and cycle-rooted spanning trees}

Pemantle [54] showed that the branch connecting two vertices in a uniform spanning tree has the distribution of a loop-erased random walk (LERW) between them. A convenient way to see this, is to look at the Markov chain on arboresences where the root of an arborescence moves according to a random walk. By adding an outgoing edge from the root of an arborescence, one creates an oriented cycle-rooted spanning tree (CRST). Note that the loop of a CRST may be of size 2, which distinguishes it from a unicycle.

Figure 3 illustrates a Markov chain on CRSTs which couples it to our first model of dynamical loop-erased random walk. Since it can be shown that the Markov chain induces the uniform measure on spanning arborescences, we may relate $\rho$ to the ratio of CRSTs to spanning trees, which in turn can be related to $U_{1} / F_{1}$. We explained in the previous subsection how to compute this ratio for planar graphs.

If $\delta$ denotes the mean vertex degree of the graph, it follows from simple considerations on this Markov chain that $\delta \rho$ gives the mean number of neighboring ancestors of a uniformly chosen random vertex $u$ in a random spanning arborescence rooted at a vertex $s$. When the graph is vertex-regular, $\rho$ is thus the probability that the loop-erased random walk from $u$ to $s$ passes through a given neighbor $v$.

\subsection{Looping rate and sandpile density of planar lattices}

On an infinite planar periodic lattice the sandpile density and the looping rate are related $[33,47]$ by

$$
\bar{\sigma}=\frac{\delta \rho+\delta-1}{2} .
$$

Using this formula and the method for computing $\rho$ explained above, we can derive $\bar{\sigma}$. Recall however that there are some subtleties to make sense of the infinite volume measure as mentioned in the introduction. The bijection to trees is important to make sense of the infinite volume limit and claim that for periodic planar lattices there is a unique sandpile measure [27] and that the limit of $\bar{\sigma}$ on an exhaustive sequence of graphs is indeed the sandpile density of the infinite volume measure.

\footnotetext{
${ }^{2}$ For the complete graph on a large number of vertices, the asymptotics of this number is also known due to an exact recurrence relation for Tutte polynomials of complete graphs due to Gessel and rediscovered by Pak.
} 

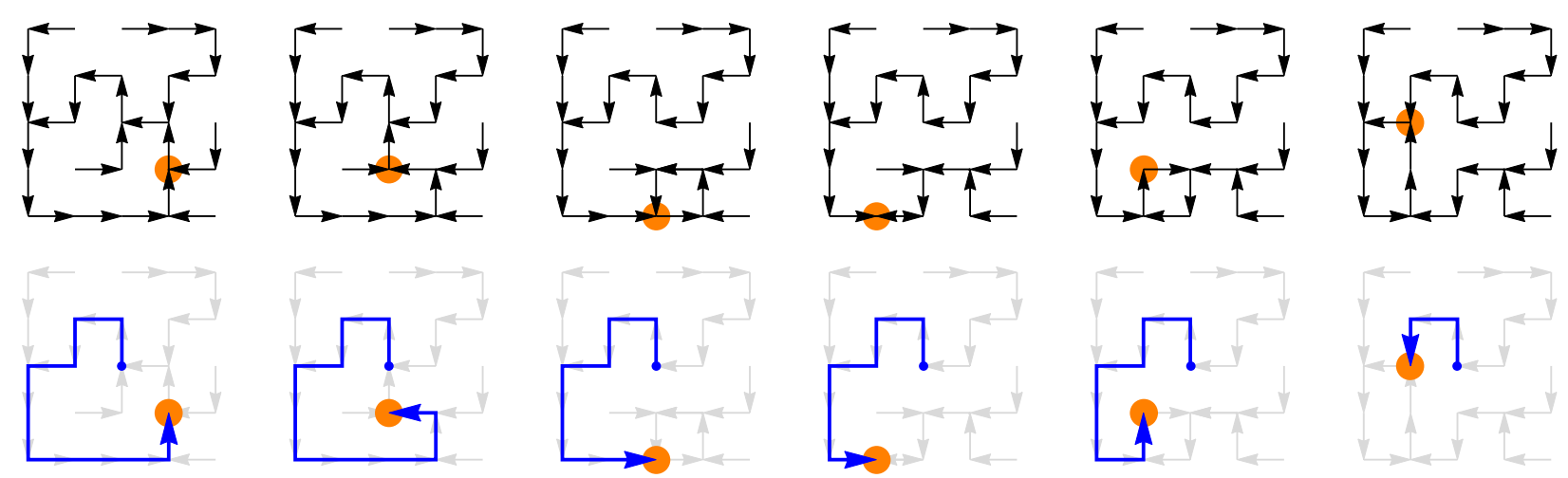

Figure 3. A sample of five consecutive steps of the Markov chain on a $5 \times 5$ square grid. The top row represents the evolution of the CRST. The bottom row highlights the simultaneous evolution of the LERW path (which starts in the center). At each step, the highlighted vertex is the current position of a simple random walk on the graph.

Another approach to the computation starts by computing $\bar{\sigma}$ and then deduces $\rho$ in the following way. Merino Lopéz [49] proved that on a finite graph the generating function for the total amount of sand of a recurrent configuration is related to the Tutte polynomial on the line $x=1$. His proof was not bijective, but later [14] provided a combinatorial proof based on one of the bijections to spanning trees mentioned above.

A straightforward computation starting from Merino Lopéz's formula relates $\bar{\sigma}$ to the ratio of unicycle to trees, which we know how to compute using the ratio $F_{2} / F_{1}$ for the dual graph. In particular for one of the three regular planar lattices, letting $\delta$ be the vertex-degree, and $\delta_{*}$ the vertex-degree of the dual lattice, we have $A_{u, v}=1 / \delta$ by symmetry, and we find

$$
\bar{\sigma}=\frac{\delta}{2}\left(1+\frac{1}{\delta_{*}^{2}}\right)
$$

giving $\bar{\sigma}=17 / 8,10 / 3$, and $37 / 24$, for the square $\left(\delta=\delta^{*}=4\right)$, triangular $\left(\delta=6, \delta^{*}=3\right)$, and honeycomb $\left(\delta=3, \delta^{*}=6\right)$ lattices, respectively (as in [39]). Using (2), the looping rate for the same lattices is found to be $\rho=5 / 16,5 / 18$, and $13 / 36$, respectively.

It is remarkable a priori that the values should all be rational. However, this is not a general fact, and for example the sandpile density of the square-octagon lattice is transcendental. More explicit values for sandpile densities are given in [33].

\subsection{Moments of the area covered by a wave of toppling}

From [25] we know that avalanches decompose into waves of topplings which are related to uniformly random two-component forests. Here again, by duality this measure is equivalent for a planar graph to the uniform measure on spanning unicycle which we now consider.

The expected length of its cycle is easily seen to be $F_{1}\left(\mathcal{G}^{*}\right) / F_{2}\left(\mathcal{G}^{*}\right)(|E|-|V|+1)$, where $\mathcal{G}^{*}$ is the planar dual of $\mathcal{G}$. In the case of a sequence of graphs exhausting the square grid, this quantity tends to 8 . The higher moments of the length are currently not tractable but moments of the area of the cycle can be computed.

For any planar simply-connected domain $D \neq \mathbb{C}$, let $\mathrm{g}_{\mathrm{D}}$ be the Green function on $D$; that is, $\mathrm{g}_{\mathrm{D}}(z, w)=$ $-1 /(2 \pi) \log \left|\varphi_{z}(w)\right|$ for any conformal map $\varphi_{z}$ sending $D$ to the unit disk and mapping $z$ to 0 .

Theorem 1.1 ( [31]). Let $D$ be a simply-connected bounded domain in the plane and $\mathcal{G}_{n}=\left(V_{n}, E_{n}\right)$ a sequence of finite connected graphs embedded in such a way that the domains $D_{n}:=\mathcal{G}_{n} / n$ approximate $D$ for the Hausdorff distance. Assume that simple random walk on $D_{n}$ converges to Brownian motion on $D$. Let $A_{n}$ be the combinatorial area of the cycle of a uniform unicycle on $\mathcal{G}_{n}$. We have $\mathbb{E}\left[A_{n}\right] \sim 2 / \pi \log \left|V_{n}\right|$ and $\mathbb{E}\left[A_{n}^{2}\right] \sim C(D)\left|D \| V_{n}\right|$, 
where

$$
C(D)=\frac{8}{|D|^{2}} \int_{D \times D} \mathrm{~g}_{\mathrm{D}}(z, w)|d z|^{2}|d w|^{2},
$$

is 8 times the average of the Green function $\mathrm{g}_{\mathrm{D}}$ on $D$ (here $|d z|^{2}|d w|^{2}$ is the Lebesgue measure on $\mathbb{C}^{2}$ ).

The constant $C(D)$ has at least two interesting interpretations. Probabilists will recognize the expected exit time of Brownian motion started at a uniform point inside the domain. Material scientists will recognize that $|D|^{2} C(D)=2 P(D)$, where $P(D)$ is the torsional rigidity of a cylinder beam of cross-section $D$. Among all bounded domains, the disk is the one which maximises $C(D)$ for which it is $1 / \pi$. This follows either from a rearrangement inequality for Gaussian processes (Brascamp-Lieb-Luttinger rearrangement inequalities [10]) using the Brownian motion interpretation, or by a Steiner reflection argument due to Pólya [56] using the torsional rigidity interpretation. As an example, for a rectangle $D$ of size $1 \times \tau$, the constant is $C(D)=$ $512 \beta(\tau) / \pi^{6}$ where

$$
\beta(\tau)=\sum_{k, \ell \geq 1, \text { both odd }} \frac{\tau}{k^{2} \ell^{2}\left(k^{2}+\tau^{2} \ell^{2}\right)} .
$$

Higher moments of the area may also be computed although the current proof known is more involved than for the first two moments. The growth rate obtained in the following theorem is consistent with a power law decay for the area distribution and the predicted critical exponents.

Theorem 1.2 ( [31]). Consider the same assumptions as in the previous theorem. For any $k \geq 1$, there exists $C_{k}(D)>0$ such that, as $n \rightarrow \infty$,

$$
\mathbb{E}\left(A_{n}^{k}\right) \sim C_{k}(D)\left|V_{n}\right|^{k-1} .
$$

This result backs the prediction that, asymptotically, $\mathbb{P}\left(A_{n}>x\right) \asymp 1 / x$. Interestingly, the analog of Theorem 1.2 turns out to be much more precise and easier to prove on a "random lattice". This was achieved by Sun and Wilson [60] who considered the setup of a jointly random pair consisting of a random planar rooted map and a spanning unicycle of it. The probability distribution of this pair is such that the marginal on random planar rooted maps is proportional to the number of its spanning unicycles. Under this annealed distribution, they were able to compute the asymptotic joint law of the length and area of the spanning unicycle and as an application they concluded that the sandpile density on a large random planar map (in the annealed distribution where the sink is chosen uniformly at random) converges to $5 / 2$.

\subsection{Height correlations}

The problem of determining height correlations in the sandpile model is much harder than merely computing $\bar{\sigma}$. It was solved by Priezzhev [57] for the square grid $\mathbb{Z}^{2}$ (modulo the computation of the sandpile density which at that time was expressed as a complicated integral whose value could only be estimated by a computer) and his results were further improved with the help of other authors (see $[16,26]$ for historical remarks). The long-range correlations of heights at two distant sites were shown or conjectured to be of the form: a power of the logarithm of the distance divided by the fourth power of the distance. Ruelle further argued in [58] that the abelian sandpile model is an example of a lattice logarithmic conformal field theory.

A more complete and robust computation of all correlations for planar graphs has been achieved by Wilson [63] using techniques of Kenyon and Wilson [39]. This machinery is based on representation theory (see Section 2.2) and allows them to compute the probability that the loop-erased random walk path to infinity goes through any particular edge or vertex. This is also related to the combinatorics of topological events for groves, see [37].

There is a special class of sub-configurations, called minimal patterns, that can be treated using the bijection with trees, for in this case correlations for the sandpile do correspond to local events for trees as proved in [27] and one may use standard result of [11] to compute uniform spanning tree correlations. In [34] with Wei Wu, we used this fact to show that the fields of occurence of such sub-configurations behave like white noise in the scaling limit (extending the result of [18]). 


\section{Geometric topology on Finite GRAPhS}

In this section $\mathcal{G}=(V, E)$ is again a finite connected (non-necessarily planar) graph. A cycle-rooted spanning forest (CRSF) of a graph is a spanning subgraph each component of which contains a unique cycle (see Figure 4). We say that a CRSF is oriented if each of its cycle has a prefered orientation. Given a subset of vertices $S$ called the boundary, an essential CRSF with Dirichlet boundary conditions at $S$ is a spanning subgraph each of whose component disjoint from $S$ is a unicycle, and where all components intersecting $S$ are trees.

It will become soon clear why CRSFs are related to the loop-erased random walk. Furthermore, CRSFs exhibit nice features due to their rich algebraic structure. In this section, we explain this form of "integrability" which makes the model well-suited to analysis (unlike many more realistic models of statistical physics). We first present an exact sampling algorithm, then a generalized version of the Matrix-Tree Theorem, and finally a theorem

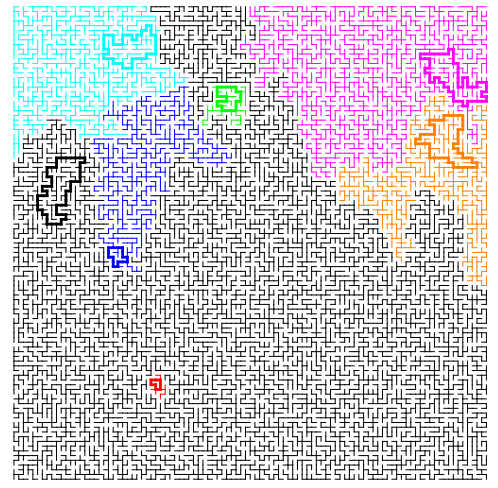

FIGURE 4. CRSF on a square grid about the determinantal nature of random CRSFs processes (implying e.g. their negative association).

\subsection{Sampling algorithm}

If $\alpha$ is a weight function on oriented loops taking values in $[0,1]$, and given any oriented essential CRSF $\Gamma$, we define $\alpha(\Gamma)=\prod_{\gamma} \alpha(\gamma)$. To sample an oriented CRSF $\Gamma$ with probability $\mathbb{P}_{\alpha}$ proportional to $\alpha(\Gamma)$, one may proceed as follows.

We define the following subroutine $R_{\alpha}(w, S)$, where $w$ is a vertex and $S$ a subgraph of $\mathcal{G}$, which outputs a random subgraph containing $S$. We first define it for $S$ nonempty. Consider the full trajectory of a random walk started at $w$ and stopped when it hits $S$. Now consider all loops that have been created chronologically and for all of them, flip an independent coin with bias $1-\alpha(\gamma)$ towards heads. If none of these coins shows heads, add the loop-erasure of the path to $S$ and output this subgraph. Otherwise, consider the first loop whose coin landed on heads and add to $S$ the loop-erasure of the trajectory until this loop is reached along with the loop itself. This creates a new connected component. If $S$ is empty, perform the random walk and flip coins as you go until eventually a coin flip comes up heads (this almost surely will happen if $\alpha(\gamma)>0$ for some $\gamma$ ). Then output the loop-erasure of this trajectory along with the last loop created.

The algorithm $\mathcal{A}_{\alpha}$ is now the following: set $X_{0}=S$ and $w_{0}=w$, where $w$ is some vertex in the complement of $S$. Recursively define $X_{n+1}=R_{\alpha}\left(w_{n}, X_{n}\right)$ and $w_{n+1}$ to be any vertex in the complement of $X_{n+1}$. Stop when $X_{n}$ contains all vertices of the graph and $w_{n}$ cannot be defined. By construction, $X_{n}$ is now an essential oriented CRSF with Dirichlet boundary conditions at $S$.

Theorem 2.1 ( [30]). The algorithm $\mathcal{A}_{\alpha}$ samples a CRSF distributed according to $\mathbb{P}_{\alpha}$.

This generalizes Wilson's algorithm [62] for sampling uniform spanning trees.

\subsection{Representation theory}

We present here a simple yet powerful technology which was introduced by Kenyon in the context of twodimensional integrable statistical physics [36]. This technology is so versatile that it is encountered in many guises in different fields. In its appearance in Forman's work [21] it seems to have been motivated by the study of symbolic dynamics and of discrete analogs to analytic torsion. And similar ideas have been used in lattice gauge theory, combinatorics of coloured graphs, geometric topology, etc.

A special type of weight function $\alpha$ arises from characters of certain representations of the fundamental group of the graph. Given a group $\mathbb{A}$ called the gauge group, a connection is the data of $g_{u, v} \in \mathbb{A}$ for each directed edge, satisfying $g_{u, v}=g_{v, u}^{-1}$. The holonomy around a loop $\gamma$ is the product of the $g$ 's around the cycle and we denote it $g_{\gamma}$. A gauge transformation is the action by conjugation on a connection $g$ of a vector of group elements $\left(j_{v}\right)_{v \in V}$ as follows: $(j \cdot g)_{u, v}=j_{u} g_{u, v} j_{v}$. 
Let $\mathcal{M}$ be the space of connections modulo gauge transformation. When the gauge group $\mathbb{A}$ is abelian, $\mathcal{M}$ has a cohomological interpretation as the cohomology group $H^{1}(\mathcal{G}, \mathbb{A})$, which by the universal coefficient theorem (see e.g. [23, Theorem 3.2.]) is isomorphic to $\operatorname{Hom}\left(H_{1}(\mathcal{G}), \mathbb{A}\right)$. When the group $\mathbb{A}$ is $\mathrm{U}(1)$, we can furthermore interpret this group as the Pontryagin dual (group of characters) of $H_{1}(\mathcal{G}, \mathbb{Z})$.

Given a connection $g$, define the twisted Laplacian by $\Delta^{g}=\Delta \otimes g$. Its determinant is a gauge invariant (that is, a function on $\mathcal{M}$ ) and it may be computed via the following theorem.

Theorem 2.2 (Forman [21] for $\mathbb{A}=\mathrm{U}(1)$; Kenyon [36] for $\mathbb{A}=\mathrm{SL}_{2}(\mathbb{C})$ ). When $\mathbb{A}$ is $\mathrm{U}(1)$ or the group of unimodular complex quaternions (isomorphic to $\mathrm{SL}_{2}(\mathbb{C})$ ), the determinant ${ }^{3}$ of the twisted Laplacian has the following combinatorial interpretation:

$$
\operatorname{det} \Delta_{\widehat{S}}^{g}=\sum_{C R S F} \prod_{\gamma} 2\left(1-\Re\left[g_{\gamma}\right]\right)
$$

where the LHS is the minor of $\Delta^{g}$ corresponding to the erasure of rows and columns indexed by $S$, and where the sum in the RHS is over all essential CRSFs with Dirichlet boundary conditions at $S$, and the product is over all cycles of a given CRSF.

Note that this is a generalization of the classical matrix-tree theorem of Kirchhoff [40]. Indeed, by specializing to the trivial connection $g=1$, the theorem equates the number of spanning trees rooted on the boundary $S$ and the minor of the Laplacian, where rows and columns indexed by boundary vertices have been erased.

\subsection{Proof of Theorems 2.1 and 2.2}

There are several proofs of Theorem 2.2, see [21,29,36]. We present yet another one, based on the algorithm presented above, which works in the special case where $g$ is a connection such that the weight $\alpha(\gamma)=1-\Re\left[g_{\gamma}\right]$ is no greater than 1 for any simple closed curve $\gamma$ (note that since the condition is only on simple curves, such connections do exist). It is based on an idea of Lawler in the classical $(g=1)$ case (see e.g. [46, Exercise 34 page 81]).

For any subgraph $F$, write $G^{F}$ for the inverse of $\Delta_{\widehat{F}}$, the sub-matrix of $\Delta$ where rows and columns indexed by vertices of $F$ have been erased. This is the Green function with Dirichlet boundary conditions at $F$ and for any vertex $x \in V \backslash F$ the diagonal entry $G_{x, x}^{F}$ equals the total mass of random walk based loops not touching the boundary weighted by their holonomy. If such a based loop consists in $k$ simple loops in chronological order, then there are $2^{k}$ loops with the same (unoriented) trace; summing them up gives a contribution of $2^{k} \prod_{i=1}^{k}\left(1-\alpha\left(\gamma_{i}\right)\right) \mathbb{P}(\gamma)$. Hence $G_{x, x}^{F}$ is also the total mass of based loops not touching the boundary weighted by $\prod_{i=1}^{k}\left(1-\alpha\left(\gamma_{i}\right)\right)$ where $\left\{\gamma_{i}\right\}_{i=1}^{k}$ is the chronological decomposition of the loop in simple loops.

Let $F$ be a subgraph of $\mathcal{G}$. A lasso is a directed path with a loop, like in Figure 1 . Consider a lasso $\left(x_{1}, \ldots, x_{k}\right)$ where $x_{k}=x_{i}$ for some $i<k$ and write $\gamma$ for its loop. Assume the lasso is disjoint from $F$. Now conditional on $F$ being a subgraph of the random CRSF, the probability of seeing this lasso in the ouput of the algorithm $\mathcal{A}_{\alpha}$ is

$\left[\operatorname{deg}\left(x_{1}\right) G_{x_{1}, x_{1}}^{F}\right] \times \operatorname{deg}\left(x_{1}\right)^{-1} \times\left[\operatorname{deg}\left(x_{2}\right) G_{x_{2}, x_{2}}^{F \cup\left\{x_{1}\right\}}\right] \times \operatorname{deg}\left(x_{2}\right)^{-1} \cdots\left[\operatorname{deg}\left(x_{k-1}\right) G_{x_{k}, x_{k}}^{F \cup\left\{x_{1}, \ldots, x_{k-1}\right\}}\right] \times \operatorname{deg}\left(x_{k-1}\right)^{-1} \times \alpha(\gamma)$

By decomposing any CRSF in the order in which it is constructed in the algorithm, and computing the probability of occurrence of each successive lassos and branches as above, we find that the probability of any CRSF is $\operatorname{det}(G) \prod_{i} \alpha\left(\gamma_{i}\right)$. This uses the simple fact that for any ordering $V=\left\{y_{1}, \ldots, y_{n}\right\}$ of the vertices, one has

$$
\prod_{\ell=1}^{n} G_{y_{\ell}, y_{\ell}}^{\left\{y_{1}, \ldots, y_{\ell-1}\right\}}=\operatorname{det} G
$$

\footnotetext{
${ }^{3}$ For quaternionic matrices, the determinant is defined in (9) below.
} 
which can be proved by rewriting the lef-hand side $\left(\right.$ using $\left.G_{x, x}^{F}:=\left(\Delta_{\widehat{F}}^{g}\right)_{x, x}^{-1}=\operatorname{det} \Delta_{\overparen{F \cup\{x\}}}^{g} / \operatorname{det} \Delta_{\widehat{F}}^{g}\right)$ as a telescoping product of ratios of decreasing minors of $\Delta^{g}$. This proves Theorem 2.1.

By writing that the total mass of the probability measure on CRSFs is 1, we have

$$
1=(\operatorname{det} G) \times \sum_{\Gamma \operatorname{CRSF}} \alpha(\Gamma),
$$

and Theorem 2.2 follows since $\operatorname{det} \Delta^{g}=(\operatorname{det} G)^{-1}$.

\subsection{Determinantal processes}

A determinantal point process on a finite set $\Lambda$, which we identify with $\{1, \ldots, n\}$, is a point process such that there exists an $n \times n$ matrix $K$ such that the probability that a subset $F$ belongs to a random sample is given by the principal minor $\operatorname{det} K_{F}$, where $K_{F}$ is the square submatrix of $K$ indexed by $F$.

We wish to extend this definition to quaternionic matrices. Define the conjugate of a quaternion $q=$ $a+b \mathfrak{i}+c \mathfrak{j}+d \mathfrak{k}$ to be $\bar{q}=a-b \mathfrak{i}-c \mathfrak{j}-d \mathfrak{k}$ (also when the $a, b, c, d$ are complex numbers). A quaternionic matrix is said to be self-dual if its transpose is equal to the matrix of its conjugated entries. The Qdeterminant of a quaternion self-dual matrix is defined [52] as

$$
\operatorname{det} K=\sum_{[\sigma]} \prod_{\text {cycles }}(-1)^{\left|c_{i}\right|-1} 2 \Re^{\prime}\left[K_{\gamma}\right],
$$

where the sum is over equivalence classes of permutations in $\mathfrak{S}_{n}$ sharing the same cycle decomposition modulo cyclic shift and reversal; in the product, $\Re^{\prime}=\Re / 2$ if the cycle is of length 1 and $K_{\gamma}$ denotes the product of entries of $K$ along $\gamma$.

We may now extend the above definition. A point process on $\Lambda$ is said to be Qdeterminantal if there is a self-dual matrix $K$ such that for any subset $F$, the probability that the process contains $F$ is the Qdeterminant of $K_{F}$. The main theorem of Chapter 5 of [29] is the following.

Theorem 2.3 ( [29]). A self-dual kernel is the kernel of a Qdeterminantal process if and only if its spectrum is in $[0,1]$. The number of points in any subset of $\Lambda$ is given by a sum of independent Bernoulli (which are non-measurable with respect to the process in general). Qdeterminantal processes form an intermediate class stricly containing that of Hermitian determinantal processes and strictly contained in that of Pfaffian processes.

The motivation for singling out these processes (which were already considered, but not explicitly, in Dyson and Mehta's work on random matrix ensembles) is that CRSFs weighted by connections in $\mathrm{SU}_{2}$ form an example of a natural family of such processes. More precisely, define, for any unitary connection $g$ in $\mathrm{SU}_{2}(\mathbb{C})$, the probability measure $\mathbb{P}_{g}$ on unoriented CRSFs giving a CRSF a probability proportional to the product over its cycles of $2-\operatorname{tr}\left(g_{\gamma}\right)$.

In order to formulate the following theorem, we need another definition. Let $\Omega^{0}$ be the space of quaternion valued functions on $V$ and $\Omega^{1}$ the space of 1 -forms defined over the edges of $\mathcal{G}$. The twisted Laplacian factorizes as $\Delta^{g}=d^{*} d$, where $d: \Omega^{0} \rightarrow \Omega^{1}, f \mapsto d f(e)=g_{e, e^{+}} f(e+)-g_{e, e^{-}} f(e-)$ may be thought of as a discrete covariant derivative and where $d^{*}: \Omega^{1} \rightarrow \Omega^{0}$ is the adjoint of $d$.

Theorem 2.4 ( [29]). The processes $\mathbb{P}_{g}$, seen as point processes on the set of edges of the graph, are Qdeterminantal. A kernel of $\mathbb{P}_{g}$ is given by the matrix of the orthogonal projection of $\Omega^{1}$ onto $\operatorname{im}(d)$ in a basis indexed by edges.

In the case of connections in $U(1)$, this statement was proved by Kenyon [36].

Proof. The $\mathbb{P}_{g}$-probability of a CRSF $\Gamma$ is $\operatorname{det}\left(\Delta_{\Gamma}^{g}\right) \operatorname{det}(G)=\operatorname{det}\left(\left(d G d^{*}\right)_{\Gamma}\right)$, where we used the fact that a CRSF has as many edges as vertices, and thus the corresponding matrices $d$ and $d^{*}$ restricted to $\Gamma$ are square matrices and we can invert the order of matrix multiplication inside the determinant. We also used the multiplicativity 
of the Qdeterminant for self-dual matrices. Since the process has a constant number of points and since $d G d^{*}$ is the orthogonal projection on im $(d)$, the claim follows from the general theory of determinantal processes.

One of the standard examples for a determinantal process with symmetric kernel is the uniform spanning tree measure which is the special case corresponding to $g=1$. The previous theorem considerably extends the class of such examples. Moreover the class of Qdeterminantal processes strictly contains that of symmetric determinantal processes and is therefore a new subclass of Pfaffian processes with particularly nice properties such as the negative association property, see e.g. [55].

Note that the probability measure above may also be defined in a more axiomatic way following Lyons [50, Section 2] as the matroidal measure defined by the matrix of $d$ in the canonical basis. The corresponding matroid is the so-called bicircular matroid, for which the set of CRSFs forms a graphical representation of its bases. Counting these bases for a general (in particular non-planar) graph is \#P-hard [22] but, as we see from Theorem 2.1, we can nonetheless sample uniformly from this set of bases using the algorithm $\mathcal{A}_{\alpha}$ with $\alpha(\gamma)=1 / 2$.

\subsection{Loop soups}

The partition function det $\Delta^{g}$ of the CRSF model can be seen to be the exponential of the integral, against a natural loop measure (see [46, Chapter 2]), of the holonomy of the loop with respect to $g$. This weighted measure may be used to define a generalization of the random walk loop soup defined in [45]. In joint work with Thierry Lévy, we have extended Proposition 28 of [46] to this setting, and we conclude that the loops erased in the generalised version of Wilson's algorithm, in the case of a weight function coming from a connection whose holonomy has positive real part, has the same law (up to rearrangement and concatenation) as the loops of an independent loop soup intersecting the CRSF. Furthermore, the local time of this loop soup has the same distribution as the squared modulus of the Gaussian free field associated to $\Delta^{g}$. This is an extension of Dynkin's isomorphism theorem and will be the subject, in greater generality, of a forthcoming joint work [32].

\section{RANDOM SIMPLE CLOSED CURVES ON SURFACES}

The Schramm-Loewner evolution of parameter $\kappa=2, \mathrm{SLE}_{2}$, is the scaling limit of the loop-erased random walk $[44,59]$. The main result presented in this section is the construction, achieved jointly with Richard Kenyon, of a probability measure on finite collections of $\mathrm{SLE}_{2}$-like loops on surfaces (which we may call $\mathrm{SLE}_{2}$ multiloops). The approach of our construction [30], initiated by Kenyon in [36], and later used in [17,38] in studying the scaling limit of the double-dimer model, consists in analyzing certain observables of a random loop ensemble on a surface given by functionals over the space of flat connections modulo gauge transformation.

\subsection{Representation of surface groups}

Consider a surface $\Sigma$ and a base point $x_{0}$. The character variety is the space $\mathcal{M}_{0}=\operatorname{Hom}\left(\pi_{1}\left(\Sigma, x_{0}\right), G\right) / G$ of group homomorphisms from the fundamental group of $\Sigma$ into a Lie group $G$ up to conjugation. This space has a differential-geometric interpretation as the moduli space of flat connections with gauge group $G$ modulo gauge equivalence. In order not to complicate the setup unecessarily, let us introduce a discrete representation of flat connections based on what we have seen earlier. For a graph embedded in $\Sigma$, say that a discrete connection is flat if the holonomy of a loop is trivial when that loop is contractible on $\Sigma$.

The space $L^{2}\left(\mathcal{M}_{0}\right)$ of regular functions on the character variety is of great importance and may be studied using the concept of lamination. A

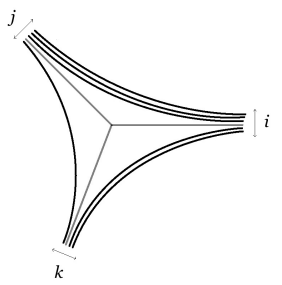

Figure 5. Local coordinates of a lamination

lamination is an isotopy equivalence classe of multicurves, that is finite families of pairwise disjoint simple closed curves embedded in $\Sigma$. Let $\mathcal{L}$ be the space of laminations. A convenient way of parametrizing $\mathcal{L}$ is to fix a trivalent surface graph and assign positive integers to its edges encoding the number of strands of a lamination 


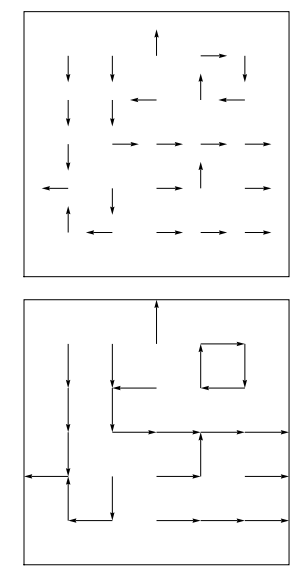

FiguRE 6. Top: random discrete vector field vanishing on the boundary; Bottom: corresponding CRSF

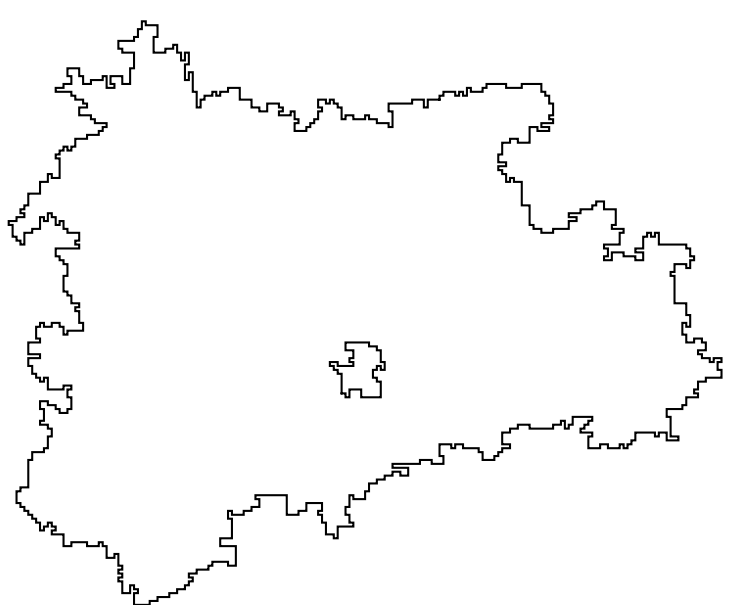

Figure 7. Periodic orbits of a random vector field

parallel to each given edge (after appropriate deformation, see Figure 5). Fock and Goncharov [20, Chapter 12] constructed a basis of $L^{2}\left(\mathcal{M}_{0}\right)$ indexed by $\mathcal{L}$.

\subsection{Topological observables}

For a flat connection $g$, Theorem 2.2 may be rewritten as

$$
\operatorname{det} \Delta^{g}=\sum_{L \in \mathcal{L}} N_{L} T_{L}(g)
$$

where $N_{L}$ is the number of CRSFs having lamination type $L$ and where the gauge-invariant $T_{L}(g)$ is defined as the product over cycles (of any representative) of $L$ of $2-\operatorname{tr}\left(g_{\gamma}\right)$. The $T_{L}$ are independent as elements on $L^{2}\left(\mathcal{M}_{0}\right)$ and we can hope to access the numbers $N_{L}$ which encode topological information on the CRSFs. These topological observables are instrumental for the proofs of the following theorems.

In the following, our probability space is the metric space $\Omega$ of multicurves up to time-reparametrization. We construct probability measures on $\Omega$ coming from the limit of measures on CRSFs on a sequence of graphs approximating $\Sigma$. By approximation, we mean that random walk converges to Brownian motion.

\subsection{Flat surfaces}

Assume the surface is not simply-connected and for any finite graph embedded in it, consider the uniform probability measure $\mathbb{P}_{\text {nonc }}$ on noncontractible CRSFs.

Theorem 3.1 ( [30]). With the above assumptions, $\mathbb{P}_{\text {nonc }}$ converges to a limiting probability measure on $\Omega$.

The proof is based on showing the convergence of some types of events for laminations which can all be expressed in terms of the Green function (or loop soup). Tightness follows from the sampling algorithm which relates the local structure of the path to that of loop-erased random walk which was shown to be a simple curve in the limit $[2,3,44,59]$.

Benoist and Dubédat [7] showed that there exists an infinite sigma-finite measure on $\mathrm{SLE}_{2}$-loops which is conformally covariant, thus answering a question of [41]. The probability measure constructed above in the case of an annulus coincides with the restriction of this measure. 


\subsection{Curved surfaces}

Define $\mathbb{P}_{\mathrm{LC}}$ to be the measure $\mathbb{P}_{g}$ on CRSFs where the discrete connection $g$ is obtained by paralleltransporting the Levi-Civita connection of the Riemannian surface along the edges of the graph.

Theorem 3.2 ( [30]). With the above assumptions, $\mathbb{P}_{\mathrm{LC}}$ converges to a limiting probability measure on $\Omega$.

Taking a limit in which the curvature tends to zero, one may study [30] the scaling limit of the measure on spanning unicycles weighted by the square of their area. This is used in the proof of Theorem 1.2 above.

In his combinatorial geometry program, Forman views CRSFs as discrete vector fields on graphs (vanishing only at boundary vertices). Figure 6 illustrates this. In view of the previous results, we may think of the scaling limit of the CRSF loops as the orbits of a random continuous flow, see Figure 7.

\section{CONCLUDing REMARKS}

Describing the scaling limit of geometrical features such as large avalanches in the two-dimensional abelian sandpile model remains a largely open problem. Dhar [15] initially made the simple observation that the expected number of topplings at a vertex $y$ resulting from the addition of an extra particle at a vertex $x$ is $G^{(s)}(x, y)=\Delta_{s}^{-1}(x, y)$ which, for a large two dimensional grid, decays as the logarithm of the Euclidean distance between $x$ and $y$. This shows that avalanches do occur on large scales. Avalanches decompose into waves of topplings whose boundaries are believed to be $\mathrm{SLE}_{2}$-type loops, and the avalanches themselves are predicted to be of multifractal type. However, no real consensus on the prediction of the corresponding critical exponents seems to have been reached to date. See [16] and references therein.

In recent years, geometrical tools from (discrete and classical) complex analysis have played a major role in understanding the scaling limit of two-dimensional critical systems like the Ising model [13]. It is not unrealistic that some of the geometrical tools we discussed above could play a similar role in the effort of understanding the critical state of the sandpile and [63] seems already to be a major step towards such an achievement.

\section{REFERENCES}

[1] Himanshu Agrawal and Deepak Dhar. Distribution of sizes of erased loops of loop-erased random walks in two and three dimensions. Phys. Rev. E, 63(5):056115, 2001.

[2] Michael Aizenman and Almut Burchard. Hölder regularity and dimension bounds for random curves. Duke Math. J., 99(3):419453, 1999.

[3] Michael Aizenman, Almut Burchard, Charles M. Newman, and David B. Wilson. Scaling limits for minimal and random spanning trees in two dimensions. Random Structures Algorithms, 15(3-4):319-367, 1999. Statistical physics methods in discrete probability, combinatorics, and theoretical computer science (Princeton, NJ, 1997).

[4] Per Bak. How nature works. Copernicus, New York, 1996. The science of self-organized criticality.

[5] Per Bak, Chao Tang, and Kurt Wiesenfeld. Self-organized criticality: An explanation of the 1/f noise. Phys. Rev. Lett., 59(4):381, 1987.

[6] Itai Benjamini, Russell Lyons, Yuval Peres, and Oded Schramm. Uniform spanning forests. Ann. Probab., 29(1):1-65, 2001.

[7] Stéphane Benoist and Julien Dubédat. An SLE_2 loop measure. ArXiv e-prints, May 2014.

[8] Olivier Bernardi. Tutte polynomial, subgraphs, orientations and sandpile model: new connections via embeddings. Electron. J. Combin., 15(1):Research Paper 109, 53, 2008.

[9] Norman L. Biggs. Chip-firing and the critical group of a graph. J. Algebraic Combin., 9(1):25-45, 1999.

[10] Herm J. Brascamp, Elliott H. Lieb, and J. M. Luttinger. A general rearrangement inequality for multiple integrals. J. Funct. Anal., 17(2):227-237, 1974.

[11] Robert Burton and Robin Pemantle. Local characteristics, entropy and limit theorems for spanning trees and domino tilings via transfer-impedances. Ann. Probab., 21(3):1329-1371, 1993.

[12] Sergio Caracciolo and Andrea Sportiello. Exact integration of height probabilities in the Abelian Sandpile model. J. Stat. Mech. Theory Exp., (9):P09013, 14, 2012.

[13] Dmitry Chelkak, Hugo Duminil-Copin, Clément Hongler, Antti Kemppainen, and Stanislav Smirnov. Convergence of Ising interfaces to Schramm's SLE curves. C. R. Math. Acad. Sci. Paris, 352(2):157-161, 2014.

[14] Robert Cori and Yvan Le Borgne. The sand-pile model and Tutte polynomials. Adv. Appl. Math., 30(1):44-52, 2003.

[15] Deepak Dhar. Self-organized critical state of sandpile automaton models. Phys. Rev. Lett., 64(14):1613-1616, 1990.

[16] Deepak Dhar. Theoretical studies of self-organized criticality. Phys. A, 369(1):29-70, 2006. 
[17] Julien Dubédat. Double dimers, conformal loop ensembles and isomonodromic deformations. ArXiv e-prints, March 2014.

[18] Maximilian Dürre. Conformal covariance of the Abelian sandpile height one field. Stochastic Process. Appl., 119(9):2725-2743, 2009.

[19] Anne Fey, Lionel Levine, and David B. Wilson. Approach to criticality in sandpiles. Phys. Rev. E, 82(3):031121, 2010.

[20] Vladimir Fock and Alexander Goncharov. Moduli spaces of local systems and higher Teichmüller theory. Publ. Math. Inst. Hautes Études Sci., (103):1-211, 2006.

[21] Robin Forman. Determinants of Laplacians on graphs. Topology, 32(1):35-46, 1993.

[22] Omer Giménez, Anna De Mier, and Marc Noy. On the number of bases of bicircular matroids. Ann. Comb., 9(1):35-45, 2005.

[23] Allen Hatcher. Algebraic topology. Cambridge University Press, Cambridge, 2002.

[24] Alexander E. Holroyd, Lionel Levine, Karola Mészáros, Yuval Peres, James Propp, and David B. Wilson. Chip-firing and rotor-routing on directed graphs. In In and out of equilibrium. 2, volume 60 of Progr. Probab., pages 331-364. Birkhäuser, Basel, 2008.

[25] Eugene V. Ivashkevich, Dmitri V. Ktitarev, and Vyatcheslav B. Priezzhev. Waves of topplings in an Abelian sandpile. Phys. A, 209(3):347-360, 1994

[26] Antal A. Járai. Sandpile models. ArXiv e-prints, January 2014.

[27] Antal A. Járai and Nicolás Werning. Minimal configurations and sandpile measures. J. Theoret. Probab., 27(1):153-167, 2014.

[28] Hang-Hyun Jo and Hyeong-Chai Jeong. Comment on "Driving sandpiles to criticality and beyond". Phys. Rev. Lett., 105(1):019601, 2010.

[29] Adrien Kassel. Laplaciens des graphes sur les surfaces et applications à la physique statistique. PhD thesis, Paris 11, 2013.

[30] Adrien Kassel and Richard Kenyon. Random curves on surfaces induced from the Laplacian determinant. arXiv preprint arXiv:1211.6974, 2012.

[31] Adrien Kassel, Richard Kenyon, and Wei Wu. Random two-component spanning forests. arXiv preprint arXiv:1203.4858, 2012. To appear in Ann. Inst. Henri Poincaré Probab. Stat.

[32] Adrien Kassel and Thierry Lévy. In preparation.

[33] Adrien Kassel and David B. Wilson. The looping rate and sandpile density of planar graphs. arXiv preprint arXiv:1402.4169, 2014. To appear in Amer. Math. Monthly.

[34] Adrien Kassel and Wei Wu. Transfer current and pattern fields in spanning trees. arXiv preprint arXiv:1312.2946, 2013. To appear in Probab. Theory Related Fields.

[35] Richard Kenyon. Long-range properties of spanning trees. J. Math. Phys., 41(3):1338, 2000.

[36] Richard Kenyon. Spanning forests and the vector bundle Laplacian. Ann. Probab., 39(5):1983-2017, 2011.

[37] Richard Kenyon. The Laplacian on planar graphs and graphs on surfaces. In Current developments in mathematics, 2011, pages 1-55. Int. Press, Somerville, MA, 2012.

[38] Richard Kenyon. Conformal invariance of loops in the double-dimer model. Comm. Math. Phys., 326(2):477-497, 2014.

[39] Richard W. Kenyon and David B. Wilson. Spanning trees of graphs on surfaces and the intensity of loop-erased random walk on $\mathbb{Z}^{2}$. arXiv preprint arXiv:1107.3377, 2011. To appear in J. Amer. Math. Soc.

[40] Gustav Kirchhoff. Über die Aufösung der Gleichungen, auf welche man bei der Untersuchung der linearen Vertheilung galvanischer Ströme geführt wird. Annalen der Physik, 148(12):497-508, 1847.

[41] Maxim Kontsevich and Yuri Suhov. On Malliavin measures, SLE, and CFT. Proceedings of the Steklov Institute of Mathematics, 258(1):100-146, 2007.

[42] Gregory F. Lawler. Loop-erased random walk. In Perplexing problems in probability, pages 197-217. Springer, 1999.

[43] Gregory F. Lawler. Intersections of random walks. Modern Birkhäuser Classics. Birkhäuser/Springer, New York, 2013. Reprint of the 1996 edition.

[44] Gregory F. Lawler, Oded Schramm, and Wendelin Werner. Conformal invariance of planar loop-erased random walks and uniform spanning trees. In Selected Works of Oded Schramm, pages 931-987. Springer, 2011.

[45] Gregory F. Lawler and José Trujillo Ferreras. Random walk loop soup. Trans. Amer. Math. Soc., 359(2):767-787, 2007.

[46] Yves Le Jan. Markov paths, loops and fields, volume 2026 of Lecture Notes in Mathematics. Springer, Heidelberg, 2011. Lectures from the 38th Probability Summer School held in Saint-Flour, 2008, École d'Été de Probabilités de Saint-Flour. [Saint-Flour Probability Summer School].

[47] Lionel Levine and Yuval Peres. The looping constant of $\mathbb{Z}^{d}$. Random Structures \& Algorithms, 45(1):1-13, 2014.

[48] C. J. Liu and Yutze Chow. Enumeration of forests in a graph. Proc. Amer. Math. Soc., 83(3):659-662, 1981.

[49] Criel Merino López. Chip firing and the Tutte polynomial. Ann. Comb., 1(1):253-259, 1997.

[50] Russell Lyons. Determinantal probability measures. Publ. Math. Inst. Hautes Études Sci., 98(1):167-212, 2003.

[51] Benoît B. Mandelbrot. The fractal geometry of nature. Macmillan, 1983.

[52] Madan Lal Mehta. Random matrices, volume 142 of Pure and Applied Mathematics (Amsterdam). Elsevier/Academic Press, Amsterdam, third edition, 2004.

[53] Wendy Myrvold. Counting $k$-component forests of a graph. Networks, 22(7):647-652, 1992.

[54] Robin Pemantle. Choosing a spanning tree for the integer lattice uniformly. Ann. Probab., 19(4):1559-1574, 1991.

[55] Robin Pemantle. Towards a theory of negative dependence. J. Math. Phys., 41(3):1371-1390, 2000. Probabilistic techniques in equilibrium and nonequilibrium statistical physics. 
[56] George Pólya. Torsional rigidity, principal frequency, electrostatic capacity and symmetrization. Quart. Appl. Math., 6:267-277, 1948.

[57] Vyatcheslav B. Priezzhev. Structure of two-dimensional sandpile. I. Height probabilities. J. Stat. Phys., 74(5-6):955-979, 1994.

[58] Philippe Ruelle. Logarithmic conformal invariance in the Abelian sandpile model. J. Phys. A, 46(49):494014, $39,2013$.

[59] Oded Schramm. Scaling limits of loop-erased random walks and uniform spanning trees. Israel J. Math., 118(1):221-288, 2000.

[60] Xin Sun and David B. Wilson. Sandpiles and unicycles on random planar maps. ArXiv e-prints, June 2015.

[61] Alessandro Vespignani, Ronald Dickman, Miguel A. Muñoz, and Stefano Zapperi. Absorbing-state phase transitions in fixedenergy sandpiles. Phys. Rev. E, 62:4564-4582, Oct 2000.

[62] David B. Wilson. Generating random spanning trees more quickly than the cover time. In Proceedings of the twenty-eighth annual ACM symposium on Theory of computing, pages 296-303. ACM, 1996.

[63] David B. Wilson. Local statistics of the abelian sandpile model. manuscript, 2013. 